\title{
Hepatitis C Virus; Its Eradication from the Serum is Just Possible
}

Abdullah M. Nasrat ${ }^{1^{*}}$, Randa M. Nasrat ${ }^{2}$ and Mohammad M. Nasrat ${ }^{2}$

${ }^{1}$ Department of Surgery, Balghsoon Clinic, Jeddah, KSA

${ }^{2}$ Department of Internal Medicine, Helwan General Hospital, Helwan, Egypt

*Corresponding author: Nasrat AM, Department of Surgery, Balghsoon Clinic, Jeddah, KSA, Tel: + 966 (012) 667 3645; E-mail: abdullahalnasrat@yahoo.com

Rec date: October 19, 2015; Acc date: June 13, 2016; Pub date: June 19, 2016

Copyright: (C) 2016 Nasrat AM, et al. This is an open-access article distributed under the terms of the Creative Commons Attribution License, which permits unrestricted use, distribution, and reproduction in any medium, provided the original author and source are credited.

\begin{abstract}
The study aimed to demonstrate the effect of blood-let out (BLO) cupping therapy in eradication of Hepatitis C virus (HCV) from the serum.

HCV affects millions of people worldwide; HCV causes not only liver disease but also a significant number of extra-hepatic manifestations. HCV is a major cause of hepatocellular carcinoma which is a common life-threatening complication of longstanding infection with HCV. The clinical outcomes of chronic hepatic viral illness are determined by the rate of viral replication and the host immune response. Treatment should be directed at reduction of the viral load in serum to suppress development of complications. Interferon therapy includes serious side effects, low efficacy and the possibility of ongoing transmission and re-infection. The new directly acting antiviral drugs offer good therapeutic effect, short duration of treatment and low incidence of side effects but are costly. Cupping therapy has demonstrated effective depletion of the viral load in few hours after the cupping session and successful clinical outcomes in reasonable period of therapy.
\end{abstract}

The study included three patients with different viral loads; they had undergone repeated sessions of cupping therapy on the upper back.

The first patient, 71 years old, quit the study when his viral load dropped from 5 million units to 75000 units after two sessions and returned to interferon therapy. The second patient with viral load 750.000 units was cured after 7 sessions of cupping therapy in 15 months period. The third patient was cured after 5 sessions in 7 months period. The second and third patients were followed up for 3 and 6 months respectively and showed no recurrence.

On conclusion, cupping therapy constitutes a therapeutic revolution in hepatitis $\mathrm{C}$ viral illness treatment through massive eradication of the virus from the serum in a rapid immediate onset after the cupping session.

Keywords Chronic hepatitis C; Cupping therapy; Hepatitis C virus

\section{Introduction}

Viral hepatitis is a major public health problem; long-term sequences are the development of liver cirrhosis and hepatocellular carcinoma. Hepatitis C virus (HCV) affects millions of people worldwide. The prevalence of HCV in some countries has surpassed the prevalence of human immune deficiency virus (HIV) disease.

HCV is a hepatotropic and lymphotropic virus that causes not only liver disease but also a significant number of extra-hepatic manifestations (EHMs). Up to $74 \%$ of patients affected by HCV will have HCV-related EHMs of some severity in their life. The EHMs vary from simple cutaneous palpable purpura to complex lymphoproliferative disorders including lymphomas and immune-complex deposit diseases causing local or systemic complications [1-3].

$\mathrm{HCV}$ is a major cause of hepatocellular carcinoma. Hepatocellular carcinoma (HCC) is a common life-threatening complication of longstanding infection with $\mathrm{HCV}$, likely a consequence of the direct oncogenic activity of the virus cooperating with liver cell inflammation in transforming the liver into a mitogenic and mutagenic environment. The achievement of a sustained viral response (SVR) to interferon- based therapies has been shown to benefit the course of hepatitis $\mathrm{C}$ viral illness in terms of reduced rates of liver-related complications and mortality. Interestingly, the achievement of SVR is associated with a negligible risk of developing clinical decompensation over the years $[4,5]$.

Several EHMs are associated with chronic HCV infection. Patients with HCV may develop mixed cryoglobulinemia (MC) and its sequels ranging from cutaneous and visceral vasculitis to glomerulonephritis and $\mathrm{B}$ cell non-Hodgkin lymphoma. HCV-infected patients have increased rates of insulin resistance, diabetes, and atherosclerosis which may lead to increased cardiovascular morbidity and mortality. Neurological manifestations of HCV infection include fatigue and cognitive impairment [5].

MC is manifested by multiple systemic organ involvement mainly skin, kidneys, peripheral nerves, salivary glands, and less frequently it may cause widespread vasculitis and malignant lymphoma. Up to $3 \%$ of HCV-infected patients are affected with cryoglobulinemia of clinical significance. EHMs of $\mathrm{HCV}$ may become a major indication for treatment even in the absence of liver disease. Severe disease requires immunosuppressive or plasma exchange therapy $[3,6]$. 
The most common HCV genotypes are genotypes 1 and 3 . The clinical outcomes of chronic hepatic viral illness are determined by the viral replication and the host immune response. Treatment therefore should be directed at reduction of viral load in the serum and suppression of viral replication before development of any significant irreversible liver cell damage. HCV replication in anti-HCV positive patients varies from $45 \%$ to $90 \%$ in different studies $[7,8]$. Spontaneous elimination of the virus occurs in minority of infected individuals and cellular response directed against HCV antigens is not persistent in individuals with chronic infection. The role of HCV-specific cellular immune response would contribute to better understanding of immune-pathogenesis and may help to design an effective vaccine [9].

Interferon-based therapies were until recently the standard of care in chronic hepatitis $\mathrm{C}$ (CHC) despite their side effects. The interferonbased standard of care (SOC) includes challenges such as serious side effects, low efficacy results and ongoing transmission and re-infection. Psychiatric side effects associated with IFN- $\alpha$ therapy in $\mathrm{CHC}$ patients are the main cause of antiviral treatment discontinuation which results into a decreased rate of SVR. The most common side effect of IFN- $\alpha$ therapy is depression; major depression disorder and new-onset psychosis that may lead to suicide ideation and suicide attempts $[2,10,11]$.

Recent innovations have produced breakthrough therapies that are effective in more than 90 percent of patients. These treatments could dramatically reduce the virus prevalence but are too much costly. The new directly acting antiviral drugs offer a good therapeutic option because of their good efficacy, short duration of treatment and low incidence of side effects. These highly effective oral direct antiviral regimens could constitute a therapeutic revolution in HCV treatment because of the possibility of eliminating the virus $[7,11,12]$.

Blood-let out (BLO) cupping therapy has been recently demonstrated as an effective sero-clearance therapeutic measure that has been proved effective in viral illness as concerns elimination of the undesired elements from the circulation and consequently depletion of the viral load, control of viral replication and stop of progress into complications. At the same time, augmentation of the individual immunity due to sero-clearance gives the body a chance to overcome and eradicate the virus and achieve clinical cure. Interestingly, Cupping therapy in viral illness is dramatic and revolutionary as being simple, safe, effective and costless $[13,14]$.

\section{Methods}

\section{Aim}

Demonstration of the sero-clearance effect of BLO cupping therapy in eradication of HCV from the serum and its therapeutic influence on the clinical outcomes.

\section{Design and setting}

Multiple clinical-case report study done in Balghsoon Clinics in Jeddah/Saud Arabia during May 2013-May 2015.

\section{Patients and methods}

The study included three male patients; the first patient was 71 years old on interferon therapy, he was the father in law of a clinical pathology university professor, he referred the patient to undergo cupping therapy as his viral load was 5 million units with complete failure to respond to therapy. The other two patients were younger adults aged 35 and 31 years with viral loads 750.000 and 75000 units respectively, both of them quit interferon therapy because of side effects and the second one was planning to start oral direct antiviral therapy before joining the study.

The patients were scheduled for cupping therapy sessions on the upper back once monthly for three months followed by a session every two months for three successive sessions, then a session every three months for three successive sessions. If the sessions had to be further repeated due to non-eradication of the virus it would have been six monthly for three successive sessions then once yearly only in order to maintain low levels of the virus in the serum if ever present until clinical cure is achieved.

BLO cupping therapy is a traditional therapy that could include fundamental cure which is performed by superficial skin scratching on the skin followed by suction of trapped blood from within the tissues in the interstitial space via the traditional suction cups. This traditional therapy can be described as "functional modified multiple mini fasciotomy" $[13,14]$.

\section{Results}

The first patient had cupping sessions twice with one month space between them, his viral load dropped to 75000 units; a result which was amazing to the university pathologist as his patient's huge viral load was constantly resistant to antiviral therapy, the patient then quit the study and returned back to antiviral treatment with no more news from him.

The other two patients continued the study until they were clinically cured; it is worthy to mention that since the first session their viral load in the serum, when tested the following morning, it reaches undetectable or low detectable levels. Of course, the virus slowly builds up later in the serum from the tissues but a process of continued gradual eradication of the virus from serum goes on with revision of cupping sessions.

The first of them, who started the study with viral load 750.000 units, has had seven sessions in 15 months period (three monthly sessions, three every two month each and one six month later) in order to reach clinical cure. He was followed up for three months only, he showed no recurrence during this period but did not show up after.

The second patient, who started the study with viral load 75000 units, has had five sessions in 7 months duration (three monthly sessions and two every two month each) and he was clinically cured with follow up for six months showing no recurrence.

Figure 1 shows the digital color view of venous blood samples taken from patients with HCV before and the morning after the first cupping session.

\section{Ethical Considerations}

An informed signed consent was taken from all patients, they were made aware about safety of the procedure of cupping therapy and they were free to quit the study whenever they like. Patients who were on medications were able to follow their regular treatments. 


(before)
(after)
Figure 1: Digital color view of venous blood samples taken from
patients with HCV before and the morning after the first cupping
session.

\section{Discussion}

The clinical outcomes of chronic hepatic viral illness are determined by the rate of viral replication and the host immune response. Treatment therefore should be directed at suppression of the viral replication by reduction of the viral load in serum in order to suppress development of any significant irreversible complications $[7,8]$.

Interferon therapy includes serious side effects, low efficacy and the possibility of ongoing transmission and re-infection [2,10]. The new directly acting antiviral drugs offer good therapeutic effect, short duration of treatment and low incidence of side effects but are very costly [7,11]. BLO cupping therapy has demonstrated effective depletion of the viral load in few hours and successful clinical outcomes in reasonable period of therapy [14].

According to experimental evidences, cupping BLO therapy is a traditional procedure talented for sero-clearance or elimination of the undesired elements from the circulation. Suction in cupping BLO works specifically on the blood trapped within the interstitial space and is not deriving blood from the circulation as documented by the finding that cupping suction reaches a point where blood-letting out stops whatever the suction is. Furthermore, patients under anticoagulants do not need to stop these anticoagulants before undergoing cupping for fear of bleeding as these medicines run and function inside the circulation and should have no influence on the blood trapped within the tissues. Hence, the blood which is let-out during cupping therapy actually still clots faster and stronger than any other blood as if these anticoagulants are not there; this should be a definite observational document that the blood removed during cupping is derived from within the tissues and not from the circulation. Revision of cupping suction few hours later in the same area of previous cupping reveals blood out again; the source of this new blood is none but the circulation, meaning that the circulation has sacrificed some of its elements into the interstitial space at the area of cupping simply because of being undesired consisting of abnormalshaped red blood cells which has been documented via microscopic examination; that is definitely a sero-clearance. Sero-clearance is being due to the effect of histamine release at the scratching sites which attracts the circulation to the area of cupping resembling a sort of selective pooling of blood in this area. In addition, there is production of nitric oxide (NO) due to the act of repeated suction with consequent micro-capillary dilatation in an area which has been already selectively pooled with blood leading ultimately to elimination or filtration of the circulating undesired elements and fall of the undesired old red blood cells out of the circulation $[13,14]$.
The biologic circulatory activity which takes place within a short while after the cupping procedure can be referred to as "highly selective pooling of the whole circulation within a localized sector of the capillary bed over a limited interval'. The pooling of circulation is related to histamine release at the scratch sites, while fall of the undesired spherical red blood cells out of the circulation is due to the influence of endothelial-derived NO production caused by the act of repeated suction. In normal situations, the biconcave healthy red cells circulate through the micro-capillary bed smoothly from the arterial to the venous side, while the spherical unhealthy red cells have to get squeezed to the size of micro-capillaries in order to go through the narrow capillary bed under the influence of blood pressure; these cells recoil or regain their spherical shape after they pass beyond the narrow vessels. In case of micro-capillary dilation, the matter is not much different for the healthy cells, whereas the unhealthy red cells undergo less squeeze to an ovoid oblong shape that recoils and gains a bluntshaped distal end on reaching the dilated potential micro-capillary arterio-venous space due to the influence of NO; therefore, these cells bulge and get stuck at the junction space restoring most of their spherical shape becoming unable to continue through the venous end of the capillary junction and accordingly fall into the interstitial space constituting the blood that is supposed to be withdrawn in the future cupping therapy session [14].

Scratching in cupping procedures is minutely tiny and extremely superficial touching even no micro-capillaries and the blood which is let-out during cupping procedure is blood trapped within the tissues that moves towards the cups in response to the effect of suction. There always appears ecchymosed skin at the site of cupping suction which does not disappear after re-scratching of the ecchymosed areas and repeat suction denoting its congested nature within the microcapillaries and further confirming that scratching in cupping procedures does not injure even micro-capillaries. More-over, this is frankly declaring the intelligent influence of cupping suction on the micro-vascular endothelium leading to the valuable stimulation of the endothelial-derived NO liberation. NO is a smart biologic material inside the body, the challenge in the matter of $\mathrm{NO}$ is never its administration as the modern medicine can do, such as in employing viagra with its hazards, but the challenge is the selective approach of $\mathrm{NO}$ to the required specific targeted area which exactly happens in cupping therapy [13].

The concept of "selective pooling of the circulation within a capillary bed compartment" explains the sense of sleepiness or dizziness that accompanies or directly follows cupping procedures, confirming the biologic value of cupping therapy; same as the full stomach stealing the circulation for the purpose of the biologic digestive function and thus turning the person sleepy. It also illustrates that the maximum benefit of cupping therapy is mainly expected during the interval immediately related to the cupping session; therefore, the person should relax after cupping therapy for a couple of hours at least and gain energy from light diet and drinks which is required for the biological process exerted by the circulation; avoiding steal of the circulation towards a full stomach by a big meal or to the muscles by doing un-necessary effort. This further confirms with the fact that liberation of NO is limited to a particular interval of time. The concept of the "selective localized pooling of circulation within the capillary bed in cupping therapy" gives the definite answer for those who employ too many therapeutic cups in one session; causing undue scattering the circulation in this way, wasting the value of therapy and exhausting the patient for no much or even less benefit. It could also indicate in the meantime that the theories of Chinese points of 
acupuncture and the hypothesis of the energy pathway lines in cupping therapy are not standing over solid scientific grounds as cupping therapy is letting-out of blood and its biologic value lies mainly in seroclearance consequent to a highly selective pooling of the circulation in a localized capillary bed compartment.

The "concept of pooling of the circulation within a sector of the capillary bed" explains why cupping therapy should not be done for patients with sickle cell disease. Red blood cells that contain normal hemoglobin are disc shaped; this shape allows the cells to be flexible so that they can move through major, large, small and micro-capillary vessels to deliver oxygen to cells and tissues. While sickle hemoglobin is unlike normal hemoglobin as it can form stiff rods within the red cell, changing it into a crescent or sickle shape; sickle-shaped cells are not flexible. As much as sickle cells can stick to the vessel wall and stick or cluster at micro-vascular bifurcations, they can further stick and cluster together at the sites of the NO-induced micro-capillary dilated synapses (junctions) causing a blockage that slows or stops the flow of blood, therefore; oxygen cannot reach adjacent cells and tissues, or these sickle cells could fall out of the circulation during "pooling of blood within a capillary bed" that follows cupping therapy because of their abnormal shape and non-flexibility, giving the signs and symptoms of sickle cell crisis. Sticking together of sickle cells could be also precipitated during "pooling of blood within a capillary bed" due to a further reason which is change of hemodynamic aspects of blood while flowing within the dilated micro-capillaries leading to stasis or block of circulation. Accordingly, the concept of the "highly selective pooling of the circulation within a specific sector of the capillary bed over a limited interval' should be considered the most solid accepted concept that explains the mechanism of sero-clearance in cupping therapy as it can give the answer and explanation for most questions and queries in cupping therapy $[14,15]$.

Sero-clearance is a huge biological talent which is not feasible via the available clinical measures; it is an integral reason to augment the individual immunity allowing the body in turn to combat the progress of any chronic viral illness. That is how cupping therapy via seroclearance can deplete the viral load to very low levels and that is how a patient with highly improved individual immunity due to the improved quality of oxygen and nutrient carrying capacity of blood can have the chance to overcome a virus in its very low or undetectable levels. The fact that revision of cupping procedure in the same place in healthy individuals reveals scanty or less amount of blood in next sessions but continues to let blood out in those with chronic illness refers to the purpose of revision of cupping sessions for the patients of this study in order to ensure continued biological sero-clearance so as to deal with the ongoing process of chronic illness. Therefore; regression of the amount of blood which is let-out during cupping therapy among patients of this study was considered a positive sign towards healing. The interstitial space, where a lot of biological processes exist, constitutes therefore the intelligent yard where BLO cupping therapy exerts its biological talents $[13,14]$.

\section{Conclusion}

Cupping BLO therapy constitutes a therapeutic revolution in hepatitis $C$ viral illness treatment through massive eradication of the virus from the serum in a rapid immediate onset after the cupping session via a huge biological process of sero-clearance leading to diminution of the viral load to extremely low detectable or undetectable levels arresting in turn progress of complications and allowing the chance for clinical cure. The concept of the "highly selective pooling of the whole circulation within a localized sector of the capillary bed over a limited interval' during cupping procedures seems standing over solid scientific grounds. The immediate accurate revision and scientific re-determination of the value of cupping therapy in the management of HCV for the purpose of its wide practical therapeutic application should be a worldwide scientific responsibility.

\section{References}

1. Stahmeyer JT, Krauth C, Bert F, Pfeiffer-Vornkahl H, Alshuth U, et al. (2016) Costs and outcomes of treating chronic hepatitis C patients in routine care - results from a nationwide multicenter trial. J Viral Hepat 23: 105-115.

2. Poonsapaya JM, Einodshofer M, Kirkham HS, Glover P, DuChane J (2015) New all oral therapy for chronic hepatitis C virus (HCV): a novel long-term cost comparison. Cost Eff Resour Alloc 13: 17.

3. Dedania B, Wu GY (2015) Dermatologic Extrahepatic Manifestations of Hepatitis C. J Clin Transl Hepatol 3: 127-133.

4. Mohlman MK, Saleh DA, Ezzat S, Abdel-Hamid M, Korba B, et al. (2015) Viral transmission risk factors in an Egyptian population with high hepatitis C prevalence. BMC Public Health 15: 1030.

5. D'Ambrosio R, Della Corte C, Colombo M (2015) Hepatocellular Carcinoma in Patients with a Sustained Response to Anti-Hepatitis C Therapy. Int J Mol Sci 16: 19698-19712.

6. Negro F, Forton D, Craxì A, Sulkowski MS, Feld JJ, et al. (2015) Extrahepatic morbidity and mortality of chronic hepatitis C. Gastroenterology 149: 1345-1360.

7. Zampino R, Coppola N, Sagnelli C, Di Caprio G, Sagnelli E (2015) Hepatitis $\mathrm{C}$ virus infection and prisoners: Epidemiology, outcome and treatment. World J Hepatol 7: 2323-2330.

8. Pramoolsinsup C (2002) Management of viral hepatitis B. J Gastroenterol Hepatol 17: S125-145.

9. Kazmierczak J, Caraballo Cortes K, Bukowska-Osko I (2015) Virusspecific cellular response in hepatitis $C$ virus infection. Arch mmunol Ther Exp 64: 101-110.

10. Lucaciu LA, Dumitrascu DL (2015) Depression and suicide ideation $n$ chronic hepatitis $\mathrm{C}$ patients untreated and treated with interferon: prevalence prevention and treatment. Ann Gastroenterol 28: 440-447.

11. Van Nuys K, Brookmeyer R, Chou JW, Dreyfus D, Dieterich D, et al. (2015) Broad Hepatitis C treatment scenarios return substantial health gains, but capacity is a concern. Health Aff (Millwood) 34: 1666-1674.

12. Cousien A, Tran VC, Deuffic-Burban S (2015) Hepatitis C treatment as prevention of viral transmission and liver-related morbidity in persons who inject drugs. Hepatology Review 63: 1090-1101.

13. Nasrat AM (2010) Role of blood-let out cupping therapy in taming the wild hepatitis B Virus. The International Congress of Aesthetic Dermatology and Preventive medicine, Paris.

14. Nasrat AM, Nasrat SAM, Nasrat RM (2015) Role of blood-let out cupping therapy in taming the wild hepatitis B Virus. Int J Recent Sci Res 6: 5049-5051.

15. MacCallum RN, Lynch EC, Hellums JD, Alfrey CP Jr (1975) Fragility of abnormal erythrocytes evaluated by response to shear stress. J Lab Clin Med 85: 67-74. 